\title{
LA ENSEÑANZA DEL DERECHO A LA CIUDAD EN LA FORMACIÓN DE PROFESIONALES DEL DERECHO
}

\author{
Teaching the right to the city in the training of legal professionals
}

Recibido: 15 de abril de 2021

Aceptado: 12 de julio de 2021

MSc. Ana Rosa Aguilera Rodríguez

Profesora de Derecho. Coordinadora de la Carrera

rosana@ult.edu.cu

Universidad de Las Tunas, Cuba

\section{RESUMEN}

Con el objetivo de fundamentar la necesidad de incluir la enseñanza relativa al derecho a la ciudad en la formación de los profesionales del Derecho, en aras de contribuir a su mayor integralidad, se realiza el presente artículo. Se enfatiza en Cuba, a partir de las peculiaridades que caracteriza a los planes de estudio de esta Carrera. Se tienen en cuenta las ventajas que ofrece la adquisición de una cultura jurídica sobre él, en la formación integral del jurista para el ejercicio de la profesión, fundamentalmente en esferas de actuación relacionadas con el hábitat, el ordenamiento territorial y el urbanismo. Se emplea la revisión bibliográfica como técnica investigativa, acompañada de métodos de uso frecuente en investigaciones científico- teóricas e investigaciones jurídicas. Finalmente se concluye cómo la inclusión de la enseñanza de este derecho, en el currículo que actualmente se desarrolla en el país, contribuye fehacientemente a su perfeccionamiento.

\section{PALABRAS CLAVE}

ordenamiento territorial, urbanismo, asignatura optativa.

\begin{abstract}
With the aim of substantiating the need to include teaching related to the right to the city in the training of legal professionals, in order to contribute to its greater comprehensiveness, this article is written. Its is emphasized in Cuba, based on the peculiarities that characterize the study plans of this career. The advantages offered by the acquisition of a legal culture on it are taken into account, in the comprehensive training of the jurist for the exercise of the profession, fundamentally in spheres of action related to habitat, land use planning and urban planning. The bibliographic review is used as an investigative technique, accompanied by methods frequently used in theoretical scientific research and legal research. Finally, it is concluded how the inclusion of the teaching of this right, in the curriculum that is currently being developed I the country, reliably contributes to its improvement.
\end{abstract}

\section{KEYWORDS}

land use planning, urban planning, optional subject. 
Sumario: 1. Introducción. 2. Apuntes sobre el derecho a la ciudad y la necesidad de su inclusión en la enseñanza del Derecho en Cuba. 3. La enseñanza del derecho a la ciudad y su vínculo con la cultura jurídica en Cuba. 4. Conclusiones. 5. Bibliografía.

\section{Introducción.}

La enseñanza del Derecho en Cuba, es una de las más antiguas en la educación superior; contribuir a su perfeccionamiento es una labor loable; sobre todo, por lo que esta significa para la sociedad. Así, es continua la labor de ajuste que se realiza respecto a sus planes de estudio, siempre en correspondencia con las necesidades e intereses formativos que se requieren para el logro de un profesional integral cada vez más competente.

A partir de las flexibilidades que admite el proceso docente educativo, es posible la inclusión de asignaturas que tributen al currículo básico, propio y optativo, de una manera pertinente y oportuna; de modo que respondan a las necesidades reales de la formación. Específicamente el optativo, se caracteriza por asimilar aquellas que determine el territorio en el cual se imparte la Carrera, de acuerdo con las prioridades que primen en el mismo, tras la colegiación que al respecto se realiza, esencialmente, entre las instituciones jurídicas ${ }^{1}$ y la Universidad.

Tomando en consideración lo referido ut supra, se realiza el presente artículo, con el que se pretende fundamentar la necesidad de incluir la enseñanza relativa al derecho a la ciudad en la formación de los profesionales del Derecho, en aras de contribuir a su mayor integralidad. Para ello, se tiene como base, la posibilidad de lograrlo en una de las asignaturas optativas diseñadas en su plan del proceso docente: el Derecho Urbanístico; la que a la vez guarda estrecho vínculo con una de las previstas en el currículo básico: el Derecho Administrativo.

El derecho a la ciudad es un nuevo paradigma urbano que cada vez adquiere mayor connotación, no solo por sus valores, sino por su contenido, en virtud del cual, contribuye a hacer más extensivo lo relacionado con la calidad de vida de las personas, a aspectos tan medulares como su entorno, sea urbano o rural (Desagües y Martínez, 2012, p. 100). Desde sus primeras formulaciones, atribuidas al francés Henri Lefebvre ${ }^{2}$, a finales de la década de los '60 del siglo XX, hasta la actualidad, se advierte cómo su tratamiento transita por diversas disciplinas, correspondiéndole a las Ciencias Jurídicas, un lugar cimero por todo cuanto implica en la conformación de plataformas que le faciliten su concreción efectiva.

Son constatables también los avances en la producción académica ${ }^{3}$, a nivel internacional, desde la cual se ahonda en una variada gama de concepciones sobre él, reveladoras de cómo este derecho es reflejo del contexto y momento histórico en el que se valora. Aunque disímiles son las posiciones teóricas respecto a él, existe consenso en

\footnotetext{
${ }^{1}$ Se hace referencia, fundamentalmente a los Bufetes Colectivos, a los Tribunales Populares, a las Fiscalías, así como a las Direcciones de Justicia en sus disímiles departamentos.

2 El filósofo francés formula el concepto de "derecho a la ciudad" como un derecho a la vida urbana. Lefebvre entiende que toda producción del espacio es política, asimismo el espacio siempre es producto de la sociedad. Como producción social prevalecen los conflictos y los consensos, así que de manera democrática el ser humano tiene derecho a la construcción del espacio.

3 Se advierte una considerable producción intelectual europea, norteamericana y en menor grado, latinoamericana. Atribuyen gran valor al espacio público. Específicamente en América Latina, es significativa la producción intelectual desde las Universidades y centros independientes que se articulan con FLACSO, Clacso y diversas redes que en muchos casos crean formas de colaboración con los activistas sociales. Son muy valiosos los análisis concretos aportados entonces sobre el derecho a la ciudad.
} 
admitirlo como un instrumento para alcanzar la eficacia social de las normas relativas a la solución de las controversias urbanas; como un concepto clave para plantear cambios alternativos a los modelos de ciudad, con el enfoque jurídico requerido en el presente y de modo esencial, como alternativa a los desafíos que urge la ciudad del siglo XXI (Fauth, 2015. p.8).

En Cuba, resulta un tema poco tratado y su conocimiento teórico-práctico, escaso. No está regulado legalmente; sin embargo, se advierte cómo gran parte de su contenido se encuentra presente en disímiles disposiciones jurídicas, sobre todo, en aquellos aspectos relacionados con el hábitat, el ordenamiento territorial y el urbanismo. Cuando se valora integralmente lo que representa y se tiene en cuenta las condiciones concretas y características peculiares del país, se admite su pertinencia, así como la necesidad de que resulte abordado en una mayor medida, en pos de posible regulación futura.

Lo anteriormente referido adquiere mayor valía, si se tiene en cuenta los cimientos que para ello ofrece su actual Carta Magna (Constitución de la República de Cuba, 2019). En ella se ratifican y perfeccionan preceptos refrendados anteriormente, tocantes con la dignidad humana, la inclusión social y la igualdad; y se regulan otros como el derecho a un desarrollo integral, a un hábitat seguro y saludable, así como a una vivienda adecuada; todos, devenidos soportes esenciales para el derecho a la ciudad.

Se precisa elevar la preparación técnico y profesional relativa a este derecho, para lo cual, la academia puede resultar esencial, toda vez que es loable desde la propia enseñanza del Derecho. Ello coloca a los futuros egresados ante la posibilidad de contar con una mayor cultura jurídica en un aspecto que les amplía el horizonte, en cuanto a su desempeño en esferas de actuación relativas a materias vinculadas con el contenido de este derecho.

De ese modo, en lo que sigue, se abordan algunos aspectos generales referidos al derecho a la ciudad, a la cultura jurídica, así como al impacto que ello puede tener en el logro de un jurista que integralmente responda en mayor medida a las exigencias de la sociedad cubana actual y coadyuve, desde su preparación, a una mejor realización del Derecho.

\section{Apuntes sobre el derecho a la ciudad y la necesidad de su inclusión en la enseñanza del Derecho en Cuba.}

Para entender la necesidad de incluir el derecho a la ciudad, como temática a abordar, en la enseñanza del Derecho, es preciso tomar, como punto de partida, su concepto. Aunque en los últimos años constan avances significativos en su configuración, es una labor sumamente compleja. Desde sus orígenes, se aborda esencialmente a partir de visiones filosóficas y de la teoría social, lo que resulta en extremo útil para estructurar las discusiones, que desde la óptica jurídica, se establecen en la actualidad, sobre la base de entender esa vida urbana renovada como una ciudad donde hay goce pleno y efectivo de los derechos humanos, extendido a los asentamientos humanos en general.

Tomando como referencia las concepciones de Lefebvre (1978), son disímiles ${ }^{4}$ las que giran alrededor del derecho a la ciudad, todas en dependencia del contexto en el cual se ofrezca. La idea lefebvriana se basa en la negativa impronta dejada por el

\footnotetext{
${ }^{4}$ El concepto desarrollado por H.Lefebvre en su libro clásico Le Droit à la ville (El derecho a la ciudad) es reivindicado por voces como Jacobs y más reciente por Harvey y Borja. También vale mencionar el trabajo realizado por Poncé, Oszlak, Rolnik, Delgadillo, Capel, Bolívar, Carrión, Sugranyes, Castells, Montoya, Zapata, Slavin, Erazo, Garnier, Pírez, Mathivet, Ziccardi, Dammert, Garcia, Anduaga, Alvarado, Ugalde, entre otros.
} 
neoliberalismo, principalmente en ciudades de países con economía capitalista, en las que estas se transfiguran en una mercancía puesta a disposición de los intereses de la acumulación del capital. Para él significa el derecho a vivir en una sociedad donde todas las personas tienen la misma libertad para satisfacer sus propios deseos y donde todos reciben apoyo para hacerlo.

También es de significativo valorar en este empeño, el respaldo jurídico que recibe desde los diferentes niveles. Existe una amplia tendencia de movimientos sociales, redes, académicos, organizaciones de la sociedad civil ${ }^{5}$, organizaciones gubernamentales y no gubernamentales a nivel mundial, regional y nacional, así como varios Estados, que apuestan por este derecho como una verdadera alternativa para contrarrestar las problemáticas urbanas actuales. Tal es así, que desde el inicio del siglo XXI, se promulgan una serie de instrumentos jurídicos, que aunque unos no tienen el mismo carácter vinculante que otros, sí contribuyen todos a perfilar de una mejor manera lo que se entiende por este derecho.

En ese tenor, merecen ser citadas varias Cartas, que en común, tienen el mérito de recoger los avances, discusiones y esfuerzos globales por impulsar el derecho a la ciudad en el ordenamiento jurídico internacional. Entre ellas se encuentran: la Carta Europea de Salvaguarda de los Derechos Humanos en la Ciudad (Francia, 2000), firmada por más de 400 ciudades europeas; la Carta Mundial del Derecho a la Ciudad, propuesta por los movimientos sociales reunidos en Porto Alegre, en el I Foro Social Mundial (Brasil, 2005); la Carta de Derechos y Responsabilidades de Montreal (Canadá, 2006); la Carta de la Ciudad de México por el Derecho a la Ciudad (México, 2010); la Carta-Agenda Mundial de Derechos Humanos en la Ciudad, adoptada formalmente por el Consejo Mundial de Ciudades y Gobiernos Locales Unidos (Florencia, 2011) y la Carta de Derechos Humanos de Gwangju (Corea del Sur, 2012).

Aunque todas son relevantes, dentro de ellas vale hacer un breve detenimiento en la Carta Mundial sobre el Derecho a la Ciudad y posteriormente la Carta de la Ciudad de México por el Derecho a la Ciudad. La primera, se admite como una de las esenciales en la concepción del derecho analizado. Tiene el mérito además, de reconocer los compromisos y medidas necesarios a asumir por la sociedad civil, los gobiernos locales y nacionales, parlamentarios y organismos internacionales, quienes en aras de garantizar que todas las personas vivan dignamente en las ciudades, generan instrumentos jurídicos que buscan normar los derechos humanos en el contexto urbano.

En su propio Preámbulo, se reconoce como un instrumento dirigido a contribuir con las luchas urbanas y con el proceso de reconocimiento, en el sistema internacional de los derechos humanos, del derecho a la ciudad. Es un texto político fruto del trabajo de diversas asociaciones latinoamericanas, articulada por Habitat International Coalition. En ella, el derecho a la ciudad se conceptualiza como:

(...) el usufructo equitativo de las ciudades dentro de los principios de sustentabilidad, democracia y justicia social; es un derecho colectivo de los habitantes de las ciudades, en especial de los grupos vulnerables y desfavorecidos, que les confiere legitimidad de acción y de organización, basado en sus usos y

\footnotetext{
${ }^{5}$ Entre estas organizaciones de la sociedad civil se encuentran la Habitat International Coalition (HIC), principal organización a escala mundial que recoge después del Foro Urbano Mundial de Estambul (1996) el concepto integrador de "derecho a la ciudad", así como la Plataforma Global por el Derecho a la Ciudad.
} 
costumbres, con el objetivo de alcanzar el pleno ejercicio del derecho a un patrón de vida adecuado (Carta Mundial del Derecho a la Ciudad, 2005).

La Carta de la Ciudad de México por el Derecho a la Ciudad del 2010, también es significativa. Retoma, a la vez que examina y ensancha los principios preceptuados en la Carta Mundial por el Derecho a la Ciudad. Llama la atención cómo añade inquietudes y propuestas, generadas por varios actores, relacionados con la producción y gestión social del hábitat, la responsabilidad relativa al manejo responsable de los bienes comunes, así como el derecho al disfrute equitativo del espacio público, en sus disímiles dimensiones. Se le reconoce una articulación más jurídica del concepto; concretamente lo define como:

(...) el usufructo equitativo de las ciudades dentro de los principios de sustentabilidad, democracia, equidad y justicia social. Es un derecho colectivo de los habitantes de las ciudades, que les confiere legitimidad de acción y de organización, basado en el respeto a sus diferencias, expresiones y prácticas culturales, con el objetivo de alcanzar el pleno ejercicio del derecho a la libre autodeterminación y a un nivel de vida adecuado. El Derecho a la Ciudad es interdependiente de todos los derechos humanos internacionalmente reconocidos, concebidos integralmente, e incluye, por tanto, todos los derechos civiles, políticos, económicos, sociales, culturales y ambientales reglamentados en los tratados internacionales de derechos humanos (Carta de la Ciudad de México por el Derecho a la Ciudad, 2010, apartado 1.1.)

Por su significado, es menester también considerar, en estos apuntes sobre el derecho a la ciudad, la conferencia Hábitat III, desarrollada en Quito, Ecuador, en octubre de 2016. En ella, la concepción sobre este derecho ocupa la atención en una medida considerable. Se debaten fehacientemente varios argumentos en aras de lograr la inclusión explícita del derecho a la ciudad como cimiento de la Nueva Agenda Urbana ${ }^{6}$, alcanzando posiciones de respaldo o de oposición de los países participantes.

Finalmente se alcanza el propósito previsto; en ello ejerce una influencia notoria la movilización internacional. Es un avance significativo que su definición, así como sus principales contenidos y principios, formen parte de la Declaración de Quito. Representa esta la primera ocasión en que este concepto se incluye en una agenda internacional, firmada por los gobiernos nacionales al nivel de la Organización Nacional de Naciones Unidas. Expresamente se hace referencia a este derecho como:

(...) uso equitativo y el disfrute de las ciudades y asentamientos humanos, buscando promover la inclusión y asegurando que todos sus habitantes, de las generaciones presentes y futuras, sin discriminación de ningún tipo, puedan habitar y producir ciudades y asentamientos humanos justos, seguros, saludables, accesibles, económicos, resilientes y sostenibles, para promover la prosperidad y la calidad de vida para todos (ONU-Hábitat, 2016, párrafos 11-13).

Varios son los académicos que también contribuyen a darle forma a lo que se admite mayoritariamente como derecho a la ciudad, aunque no se desconoce la polisemia que caracteriza a esta institución jurídica. Harvey, por ejemplo, lo valora como el derecho colectivo a "cambiarnos a nosotros mismos, cambiando la ciudad" (1973), de acuerdo

\footnotetext{
${ }^{6}$ Nombre dado a la estrategia surgida de Hábitat III para los próximos 20 años, o sea, hasta el 2036.
} 
con los nexos sociales, el vínculo con la naturaleza, las tecnologías, el estilo de vida o los valores estéticos que se pretendan ensalzar.

Borja, por su parte, aporta una amplia e importante obra respecto a este derecho; lo valora como una respuesta democrática, cuyo mérito esencial se encuentra en resultar integradora de los derechos de los ciudadanos y, oportunamente, de los criterios urbanísticos que hacen posible su ejercicio, con especial énfasis en lo concebido como espacio público. Lo admite como un derecho, integrado por un cúmulo de derechos formales y materiales que conforman la ciudadanía (2012, p. 247).

Asimismo se tiene en cuenta lo expresado por Uceda, quien, tomando como base lo planteado por disímiles autores, manifiesta que el derecho a la ciudad es "el derecho de acceder por parte del individuo a todo lo que participa de la calidad de la vida urbana y también derecho a cambiar la ciudad según las necesidades y deseos de la mayoría de la población” (2017, p. 30).

Se destaca, además, Oslak. Con su obra aporta al concepto mayor extensión, pues atribuye gran valor a lo referido al derecho al espacio urbano, como elemento más delimitado para determinar particularidades y alcances con que puede exteriorizarse su ejercicio, con independencia del régimen del cual se trate, contrastándolo con el de derecho a la ciudad. (2018, p. 8).

En los finales de la segunda década del siglo XXI, vienen a colación autores como Carrión y Dammert. Ambos consideran que este derecho "(...) es un intento por modificar las condiciones "actuales" de producción de lo urbano, y generar condiciones que permitan un acceso equitativo o "justo" sobre este proceso y los bienes valiosos que produce" (2019, p. 10). De modo más reciente en el tiempo, se incorpora el criterio de Tapia, la que manifiesta que el derecho en análisis "incorpora los derechos humanos y los entiende como un sistema de derechos para toda la población en sus territorios" (2020, p. $3)$.

Hasta el presente, 2021, diferentes son los espacios, sobre todo académicos, en los que se continúa profundizando teórica y prácticamente sobre el derecho a la $\operatorname{ciudad}^{7}$, desde variadas latitudes geográficas. Representa una noción cuyo contenido va mutando con el devenir del tiempo (Carrión y Dammert, 2019, p. 59). El modo en que los Estados lo reconocen, varía de un ordenamiento jurídico a otro, desde los que lo consagran en sus leyes principales, hasta los que ignoran su existencia. Lo cierto es que sobrepasa las concepciones teóricas recogidas en una serie de documentos y textos jurídicos; tiene aplicabilidad práctica, con efectos inmediatos sobre la vida de las personas.

A los efectos de este artículo, la autora lo define como aquel en virtud del cual sus titulares tienen el derecho de usar y disfrutar los beneficios que representa la ciudad en asentamientos humanos sostenibles, así como participar en la producción de los mismos de manera inclusiva, todo sobre la base de la dignidad humana, la justicia social, la igualdad, la equidad y la solidaridad, en función de lograr una mayor calidad de vida para todos.

\footnotetext{
${ }^{7}$ En tal sentido, puede referirse la red CLACSO, la que cuenta con el grupo "Desigualdades Urbanas", integrado por más de cien investigadores de distintas latitudes; su objetivo es profundizar en el debate sobre las desigualdades en la región, al centrar la atención y problematizar el espacio urbano como dimensión relevante para comprender las lógicas de producción y reproducción de las desigualdades. Asimismo, sobresale el trabajo que vienen desarrollándose por la Dra. Emanuelli, el Dr. Slavin, la Dra. Di Virgilio, el Dr. Sevilla, el Dr. J. Erazo, el Dr. Pírez, el Dr. Ponce, la Dra. Canestraro, el Dr. Santiago y el Dr. Cabrera, entre otros.
} 
Como se constata, es en un derecho social, de cuyo contenido se advierte que es inseparable de los principios en los cuales encuentra sustento, según los aspectos ya abordados, los que, en el ámbito de la ciudad y de los asentamientos humanos en general, implican que desde el Estado se accione en pos de contrarrestar las desventajas sociales, económicas, culturales y políticas, que perturban a determinados grupos de personas. De ese modo, se contribuye a erradicar la discriminación a la que son sometidos; entre ellas, las que se basan en estigmas territoriales y que afectan considerablemente al equilibrio que debe existir en ese sentido. El derecho a la ciudad, en definitiva, coadyuva al logro de la cohesión social y de la cohesión territorial. Uno de sus valores es que supone la inclusión de todos los ciudadanos en el disfrute de su contenido.

Este derecho, ofrece instrumentos jurídicos concretos tendentes a la implementación del paradigma sobre ciudades y territorios como derechos, en función de transformar los asentamientos humanos en bienes comunes y en una creación colectiva. Ello requiere mayor conocimiento sobre sus aspectos teóricos y prácticos, razón que fundamenta su necesaria inclusión como uno de las temáticas a abordar en asignaturas de la carrera de Derecho.

Al valorarlo en su integralidad es evidente la correspondencia que tiene con el contenido de asignaturas como Derecho Administrativo y Derecho Urbanístico. En el primero, se regulan aspectos medulares de la función administrativa, de lo que se deduce la amplia participación que tiene la Administración Pública en las diversas relaciones sociales que se producen en el marco de la sociedad cubana. Dentro de ello, se encuentran, por supuesto, los aspectos relacionados con el ordenamiento territorial y urbanístico, sobre todo, en lo tocante con la satisfacción de los intereses más generales de la sociedad, donde el Derecho Urbanístico, particularmente, entra a jugar un rol trascendental.

Con la incorporación del derecho a la ciudad dentro del contenido de las mencionadas asignaturas, fundamentalmente en la segunda, se presenta un nuevo enfoque para el estudio de los fenómenos urbanos, sobre todo, desde la perspectiva de su ordenación y regulación jurídica, tomando también como base el ejercicio de la actividad administrativa en ese tenor. Se contribuye así también al cumplimiento de las pautas diseñadas en el actual Plan de Estudios $\mathrm{E}^{8}$, en el que se prevé que las asignaturas que integran el mismo contribuyan en mayor medida a la formación continua y sirvan de complemento a aquellas cuestiones que desde cada disciplina se conoce son problemáticas a resolver en el eslabón de base y coadyuven a la vez a elevar la formación integral del egresado del Derecho.

\section{La enseñanza del derecho a la ciudad y su vínculo con la cultura jurídica en Cuba.}

El desconocimiento de la ley no dispensa de su cumplimiento, como reza uno de los principios jurídicos. No obstante, es innegable que el hecho de conocer lo que entraña en sus postulados, contribuye considerablemente a que se realice el Derecho. Lograr la plena realización de este es una de las aspiraciones de los disímiles ordenamientos jurídicos existentes, de los cuales no se excluye el cubano; máxime si se trata de aquella en la que prima el cumplimiento espontáneo de las normas jurídicas por parte de las personas a las cuales van dirigidas; sin dejar de reconocer que la realización del Derecho también

\footnotetext{
${ }^{8}$ Plan de estudios que se implementa desde el curso académico 2018-2019.
} 
supone la aplicación estatal u oficial de las disposiciones jurídicas, de manera compulsiva por parte de los órganos estatales competentes (Fernández, 2004).

Que se cumplan de manera natural, los objetivos y prescripciones jurídicas que ordenan la conducta social, por parte de los ciudadanos, devenidos depositarios de dichas normas, revela el carácter coercitivo del Derecho. En virtud de dicha coercitividad, los ciudadanos observan las normas jurídicas, lo que hace voluntariamente, sin detenerse a considerar las responsabilidades jurídicas en las que pueden incurrir si hacen lo contrario. Ocurre entonces un estrecho acercamiento entre el contenido de las normas jurídicas y el de las normas morales, poniendo al descubierto la función educativa del Derecho y una de las formas más auténticas de realización del Derecho, en la que no es necesaria, la intervención estatal para conseguirlo, mediante sus órganos y mecanismos coactivos (Fernández, 2004).

Esta modalidad de realización del Derecho, guarda una estrecha relación con la conciencia jurídica, y a su vez, con la cultura jurídica. El Derecho forma parte ineludible de la superestructura social y como modalidad de la conciencia social, deviene expresión concreta de la conciencia jurídica. Esta última, se integra por un conjunto de concepciones, a partir de las cuales encuentran expresión las disímiles relaciones jurídicas de una sociedad dada. A través de ella, se enuncian los conceptos, teorías y doctrinas, que históricamente hacen su aparición junto a las clases sociales y se transforman a la par de las modificaciones que tienen lugar en los regímenes económicos. Es fiel expresión de lo que se percibe como legalidad e ilegalidad, justicia e injusticia, así como deberes y obligaciones. Es vital para el Derecho; su relevancia se determina, justamente, por su nexo con el orden jurídico reconocido en la sociedad.

Vale distinguir los mecanismos de acción del Derecho y de la conciencia jurídica; mientras los primeros tienen fuerte cimiento en un complejo instrumental jurídico institucional y se sustenta en la coacción estatal, el de acción de la conciencia jurídica se basa esencialmente en cuestiones espirituales. La conciencia jurídica se revela a través de la apreciación jurídica general de los hechos sociales, evidenciada mediante la emisión de los "juicios relativos a la idea de lo justo y lo legal, del sentido del Derecho y legalidad y de la orientación volitiva concomitante de la conducta humana" (Fernández, 2004).

Muy relacionado con la conciencia jurídica, se encuentra la cultura jurídica; tanto así que a veces se confunden; sin embargo, es necesario aclarar que no significan lo mismo. Esta última, en sentido general, es el conjunto de conocimientos sobre las bases del Derecho y acerca de varias ramas del mismo, y en sus principios filosóficos, proceso histórico, doctrina y legislación positiva.

La cultura jurídica se incluye en la cultura espiritual de la sociedad, y es poseedora de una orientación aplicada y práctica. Resulta fiel expresión del valor social y del progreso del Derecho. El nivel de conocimientos jurídicos adquiridos indica de alguna manera la cultura jurídica de los ciudadanos, la que se enriquece, en el caso específico de los juristas, mediante la capacitación y superación profesional sistemática; y de los estudiantes, por la inclusión de aspectos que así lo requieran en su formación profesional. Todo ello revela, además, el grado de desarrollo de la ciencia y de la mentalidad jurídicas.

Lo cierto es que la cultura jurídica es un elemento de la conciencia jurídica. La primera aporta el plano cognoscitivo de la segunda; sin embargo, esta va más allá, aunque parte de los conocimientos que se tengan acerca del Derecho. La cultura jurídica parte del conocimiento de la normativa vigente y se enriquece con la actuación de los ciudadanos conforme a esta Ciencia, la que debe ser activa en tanto contribuya a su creación y a su realización. 
La conciencia jurídica es trascendental para la correcta aplicación de las disposiciones jurídicas; no sin razón se le reconoce cuánto favorece los cauces para la valoración a cada hecho jurídico concreto y la correcta apreciación de este por los individuos. El Derecho, influye sobre el desarrollo de la conciencia jurídica de los ciudadanos, la formación de criterios fundamentales y justos sobre los requisitos jurídicos, las relaciones jurídicas, los derechos, deberes y responsabilidades. Su papel activo se manifiesta tanto con relación a la conciencia jurídica social como con relación a la individual.

Las Ciencias Jurídicas tributan al enriquecimiento y a la concreción de la conciencia jurídica. Al mismo tiempo, la expresión de las concepciones jurídicas en las normas de Derecho, influyen notablemente al desarrollo de la conciencia jurídica. En definitiva, la noción de cultura jurídica es elemental en función de la comprensión del Derecho como fenómeno social. De hecho, este no puede apreciarse solamente desde la dimensión normativa, aunque ella implique las disposiciones jurídicas de distintos niveles, las instituciones jurídicas, así como su organización y sus procedimientos. Consecuentemente, es indispensable, tener también presente la dimensión social y valorativa del Derecho, pues a través de ellas se manifiestan las actitudes, valoraciones y creencias que sobre el fenómeno jurídico, son compartidas en una sociedad. Se le añade, además, que determinan el modo en el que las personas se relacionan con las instituciones y las normas jurídicas, tanto en su papel como juristas o como ciudadanos en general.

En Cuba, el cumplimiento de las disposiciones jurídicas, sin dejarse de apoyar en la fuerza coactiva del Estado, tiende a convertirse en un fenómeno que se sustenta en la conciencia de los ciudadanos, quienes las identifican como expresión de su propia voluntad. Vale reconocer que en el país, son de muy significativo valor los logros alcanzados en la educación y se pone especial énfasis en alcanzar una cultura general integral en la población, en la que la cultura jurídica ocupa especial posición. Una elevada cultura jurídica es la condición necesaria de la realización consciente y efectiva por cada ciudadano de su deber ante la sociedad y el Estado, posibilitando el conocimiento y estricto cumplimiento de las leyes.

La educación superior cubana asume el reto de formar profesionales del Derecho responsables, con profundas convicciones jurídicas, siendo necesario elevar la cultura jurídica en el fortalecimiento de la conciencia jurídica mediante el conocimientos de los derechos, deberes y obligaciones frente al Estado y la sociedad así como las cuestiones fundamentales de las principales normativas reguladoras de la actividad profesional y como ciudadanos en general, en la intransigencia frente a las infracciones y la lucha contra ellas y la educación en el respeto a las leyes, fortaleciéndose la legalidad socialista.

Adquirir una cultura jurídica sobre lo que representa el derecho a la ciudad, significa que los futuros profesionales del Derecho, tengan un dominio, al menos básico del mismo y coadyuven al cumplimiento de su contenido, o sea, a la realización del Derecho en ese ámbito. De esa manera, también se alcanza una mayor conciencia jurídica y se allana más el camino hacia su dimensionamiento jurídico como derecho; aún requiere de mucho trabajo y fundamentos, pero tal como se logra en otros ordenamientos jurídicos, en el cubano también puede ocurrir. De hecho, aun cuando en Cuba no se regula, no significa que se desconozca e irrespete lo que representa básicamente su contenido, pero este se encuentra disperso en varias de las disposiciones jurídicas vigentes.

El derecho a la ciudad es una alternativa útil de gran valor en la actualidad por todo cuanto puede contribuir a neutralizar las problemáticas urbanas. Lo más acertado es que se dimensione jurídicamente como derecho, lo que implica delinear sus contornos para su ejercicio efectivo, exigibilidad y defensa; establecer un punto de partida para 
implementar políticas públicas más coherentes en todos los niveles, en aras de lograr cambios estructurales cualitativamente superiores, en la forma en que se gestiona el desarrollo urbano. Significa, además, dotar a los ciudadanos de una mayor seguridad jurídica en cuanto a la posibilidad del disfrute de lo que representa su contenido, en condiciones de igualdad y de justicia social.

Varias son las acciones que se pueden realizar para conseguir que la enseñanza del derecho a la ciudad se incorpore a algunas asignaturas del currículo optativo de la carrera de Derecho, esencialmente en el Derecho Urbanístico. Para ello, pueden tenerse en cuenta determinadas pautas, útiles para proponer el alcance de un sistema de habilidades que desde la asignatura, posibiliten a los futuros egresados, sobre todo, a los que se desempeñen en esferas relacionadas con el hábitat, el ordenamiento territorial y urbanístico:

1. Modernizar el pensamiento y la práctica urbanística en Cuba desde una perspectiva integrada, y en particular, jurídica. En función de ello, urge aprovechar la experiencia internacional histórica y reciente de la vinculación entre el Derecho Urbanístico y el desarrollo de las ciudades; así como los problemas actuales de la ordenación urbana desde sí misma y desde el punto de vista de la legislación.

2. Lograr que los instrumentos de planeamiento sean la forma de presentar, evaluar y escoger la mejor alternativa para alcanzar territorios y ciudades equitativas, eficientes, sostenibles, resilientes y seguros. Deben ser un elemento de consenso de intereses y un instrumento de dirección y gestión para los gobiernos locales, con el respaldo jurídico requerido para su cumplimiento.

3. Incorporar en mayor medida a los implicados (instituciones, organismos sectoriales, entidades económicas, población) como agentes de la identificación de problemas y propuestas de soluciones, teniendo en cuenta sus conocimientos, experiencias e iniciativas, de manera que se generen conciencia y compromiso para la implementación del Plan.

4. Perfeccionar la eficacia de la gestión pública ante la creciente necesidad habitacional, el incremento del control de los recursos y la ejecución del presupuesto destinado a aspectos que garantizan la sostenibilidad urbana en las ciudades.

5. Favorecer una manera diferente de concebir el modelo de ordenamiento territorial y urbanístico para adecuarlo a los nuevos procesos políticos, sociales y económicos que lo enmarcan y con los que interactúa, incluyendo el diseño urbano.

6. Fortalecer la institucionalidad, que asegure la disciplina de las personas jurídicas $\mathrm{y}$ naturales en las transformaciones responsables, participativas y creativas de sus territorios y asentamientos humanos.

Como se aprecia, es loable incorporar la enseñanza del derecho a la ciudad en la formación de los profesionales del Derecho, quienes a su vez, también pueden contribuir a elevar la cultura jurídica de la comunidad universitaria y de la sociedad en su conjunto respecto a esta materia que cada vez cobra mayor importancia para la satisfacción de las necesidades humanas.

\section{Conclusiones.}

1. Ante las diversas problemáticas sociales vinculadas al hábitat, el ordenamiento territorial y el urbanismo, que cada vez son más evidentes a nivel mundial, del cual no se excluye el contexto cubano, es menester que desde el Derecho se ofrezcan las soluciones 
correspondientes, que garanticen la seguridad jurídica de los ciudadanos. Un paradigma urbano, que se presenta en ese tenor, es el derecho a la ciudad, el que ofrece respuestas diferentes acerca de cómo percibir los asentamientos humanos y cómo orientar las voluntades de cambio, en función de viabilizar que estos sean socialmente cada vez más sostenibles. Sin embargo, a pesar de que su connotación va en aumento y se ha ido incorporando a algunos ordenamientos jurídicos, en Cuba aún su conocimiento es escaso entre los juristas y los estudiantes de Derecho, lo que limita la visión que estos puedan tener en cuanto a los aspectos que conforman su contenido, con trascendencia a una mejor interpretación, aplicación y realización del Derecho en ese ámbito.

2. El plan de estudios de la carrera de Derecho en Cuba es flexible y se facilita el perfeccionamiento sistemático de su currículo. Ello ofrece la posibilidad de incluir en su currículo optativo, asignaturas que tributen a la preparación oportuna, sobre todo, en aquellos aspectos cuyo tratamiento no es usual, como ocurre con el derecho a la ciudad. Puede lograrse mediante la asignatura de Derecho Urbanístico. Disímiles razones sostienen la necesidad de la inclusión de este derecho como temática, del que parte de su contenido está disperso en distintas disposiciones jurídicas, aun cuando no está expresamente regulado en el ordenamiento jurídico patrio. La enseñanza relativa al derecho a la ciudad contribuye a elevar la cultura jurídica de los profesionales del Derecho y con ello, a la mayor integralidad en su formación; coadyuva a la vez, a que esta sea más pertinente, en correspondencia con las necesidades e intereses de la sociedad y con los avances científico-técnicos que se presentan en las Ciencias Jurídicas, tanto a nivel nacional como internacional.

\section{Bibliografía.}

- Asamblea Nacional del Poder Popular. (2019, 24 de febrero) Constitución de la República de Cuba. Proclamada en la Segunda Sesión Extraordinaria de la IX Legislatura de la Asamblea Nacional del Poder Popular, en el Palacio de Convenciones de La Habana, Gaceta Oficial de la República de Cuba.

- BORJA, J. (2012) "Revolución urbana y derechos ciudadanos: Claves para interpretar las contradicciones de la ciudad actual". (Tesis doctoral defendida en Barcelona).

- CARRIÓN, F. y DAMMERT GUARDIA, M. (Editores), Autores: AA.VV. (2019) "Derecho a la ciudad: una evocación de las transformaciones urbanas en América Latina". Ecuador: FLACSO.

- Carta-Agenda Mundial de Derechos Humanos en la Ciudad. (2011) Florencia: http://www.uclg-cisdp.org/sites/default/files/CISDP\%20CartaAgenda\%20Sencera_FINAL_4.pdf.

- Carta de Derechos Humanos de Gwangju. (2012) Corea del Sur.

- Carta de Derechos y Responsabilidades de Montreal. (2006) Canadá.

- Carta de la Ciudad de México por el Derecho a la Ciudad. (2010) México: http://www.porelderechoalaciudad.org.mx/.

- Carta Europea de Salvaguarda de los Derechos Humanos en la Ciudad (2000) SaintDenis - Francia: http://www.idhc.org/esp/documents/CartaEuropea.pdf.

- Carta Mundial del Derecho a la Ciudad. (2005) Porto Alegre, Brasil: http://www.onuhabitat.org/index.php?option=com_docman\&task=doc_dow nload\&gid $=50 \&$ Itemid $=3$. 
- DESAGÜES, C. y MARTÍNEZ, M. R. (2012) "Hábitat digno y Derecho a la Ciudad". Cuadernos Electrónicos $\mathrm{N}^{\mathrm{o}} 6$ Derecho a la vivienda y a la ciudad. México: UNAM, págs. 100-101.

- FAUTH, G. (2015) "Crisis urbana y derecho a la ciudad: el espacio urbano litoral de Barcelona”. Tarragona. (Tesis doctoral defendida en Universitat Rovira I Virgili.).

- FERNÁNDEZ BULTÉ, J. (2004) “Teoría del Estado y del Derecho". Cuba: Editorial Félix Varela.

- HARVEY, D. (1973) “Social Justice and the City”.

- LEFEBVRE, H. (1978) “El derecho a la ciudad”. Barcelona: Ediciones Península.

- ONU-Hábitat. (2016) Declaración de Quito, Hábitat III. Ecuador.

- OSLAK, O. (2018) "Derecho a la ciudad y derecho al espacio urbano". Voces en el Fénix, La revista del Plan Fénix 8(71). Argentina: Editorial La ciudad de los otros.

- TAPIA, M. (2020) "El derecho a la ciudad, entre el confinamiento y la utopía". Crítica Urbana. 13. A Coruña.

- UCEDA NAVAS, P. (2017) "La ciudad desequilibrada. El Derecho a la Ciudad en los barrios vulnerables de Madrid". (Tesis de doctorado defendida en Madrid). 